\title{
A DIVERGENCE-LIKE CHARACTERIZATION OF ADMISSIBLE FUNCTIONS ON DIGRAPHS
}

\author{
GEN-ICHI OSHIKIRI
}

(Received July 2, 2002, revised November 14, 2002)

\begin{abstract}
In previous papers, the author defined a notion of admissible functions for digraphs and studied its properties. The notion of admissible functions naturally comes from the study of mean curvature functions of codimension-one foliations, and admissible functions of foliated manifolds are represented by a divergence formula. In this paper, we show that the similar divergence-like formula characterizes admissible functions of digraphs.
\end{abstract}

1. Introduction. Let $D=(V(D), A(D))$ be a finite digraph, which may have loops and parallel arcs. In [7], the author defined a notion of an admissible function $f: V(D) \rightarrow \boldsymbol{R}$ and studied its properties. The notion of admissible functions naturally comes from the study of mean curvature functions of codimension-one foliations (see [9], [13]). Indeed, let $(M, \mathcal{F})$ be a codimension-one foliation $\mathcal{F}$ of a closed manifold $M$. We assume that $M$ and $\mathcal{F}$ are oriented. A smooth function $f: M \rightarrow \boldsymbol{R}$ is said to be admissible if there is a Riemannian metric $g$ on $M$ such that $-f$ is a mean curvature function of $\mathcal{F}$ with respect to the metric $g$. In this case, we have

$$
f=\operatorname{div}_{g}(N),
$$

where $N$ is the unit vector field on $M$ orthogonal to $\mathcal{F}$, and $\operatorname{div}_{g}(N)$ is the divergence of $N$ with respect to the metric $g$. From the view point of the paper [9], this situation can be interpreted by digraphs, that is, we have the following characterization of admissible functions on digraphs.

THEOREM. Let $D$ be a finite digraph. $f$ is an admissible function if and only if there is a labelling $g_{D}$ of $D$ such that $f=\delta_{g_{D}} 1,1: A(D) \rightarrow \boldsymbol{R}$ being identically 1 .

We shall give some definitions and preliminary results in Section 2, and shall prove this theorem in Section 3.

The author would like to thank the referee for his valuable comments on the first manuscript.

2. Preliminaries. Let $D=(V(D), A(D))$ be a not necessarily strict digraph, that is, $D$ may have loops and parallel arcs. In this paper, we consider only finite digraphs. An element $e=(u, v) \in A(D)$, which is an ordered pair of vertices in $V(D)$, is called an arc of $D$. The vertex $u$ (resp. $v$ ) of $e=(u, v)$ is called a tail (resp. head) and is denoted by $\alpha(e)$ (resp. $\omega(e)$ ). (see [2] for generalities on graphs). A labelling $g_{D}$ of $D$ is defined by a pair $\left(g_{V}, g_{A}\right)$ with $g_{V}: V(D) \rightarrow \boldsymbol{R}_{+}$and $g_{A}: A(D) \rightarrow \boldsymbol{R}_{+}$, where $\boldsymbol{R}_{+}=\{x \in \boldsymbol{R} \mid x>0\}$. Set

2000 Mathematics Subject Classification. Primary 05C20; Secondary 05C40, 57R30. 
$C^{0}(D)=\{f \mid f: V(D) \rightarrow \boldsymbol{R}\}$ and $C^{1}(D)=\{\phi \mid \phi: A(D) \rightarrow \boldsymbol{R}\}$. Define the coboundary operator $\delta_{g_{D}}: C^{1}(D) \rightarrow C^{0}(D)$ by

$$
\delta_{g_{D}} \phi(v)=\frac{1}{g_{V}(v)}\left(\sum_{\substack{e \in A(D) \\ \alpha(e)=v}} g_{A}(e) \phi(e)-\sum_{\substack{e^{\prime} \in A(D) \\ \omega\left(e^{\prime}\right)=v}} g_{A}\left(e^{\prime}\right) \phi\left(e^{\prime}\right)\right) .
$$

Note that if the labelling $g_{D}$ is given by $g_{V} \equiv 1 \equiv g_{A}$, then the corresponding coboundary operator $\delta_{g_{D}}$ is the usual one (cf. [1], [12]). Note also that $\delta_{g_{D}}$ of a labeled digraph $\left(D, g_{D}\right)$ corresponds to the divergence of a Riemannian manifold $(M, g)$. Indeed, define an integration of $f \in C^{0}(D)$ over a subset $W \subset V(D)$ by

$$
\int_{W} f=\sum_{v \in W} g_{V}(v) f(v) .
$$

To define an integration of $\phi \in C^{1}(D)$ for our purpose, we need some more definitions. For subsets $X, Y \subset V(D)$ with $X \cap Y=\emptyset$, we set

$$
\begin{aligned}
& \Gamma^{+}(X, Y)=\{e \in A(D) \mid \alpha(e) \in X, \omega(e) \in Y\}, \\
& \Gamma^{-}(X, Y)=\{e \in A(D) \mid \omega(e) \in X, \alpha(e) \in Y\} .
\end{aligned}
$$

If $Y=V(D) \backslash X$, then $\Gamma^{ \pm}(X, Y)$ is simply denoted by $\Gamma^{ \pm}(X)$. For a subdigraph $H \subset D$, we define the boundary $\partial H$ of $H$ by $\partial H=\Gamma^{+}(V(H)) \cup \Gamma^{-}(V(H))$, and an integration of $\phi \in C^{1}(D)$ over $\partial H$ by

$$
\int_{\partial H} \phi=\sum_{e \in \Gamma^{+}(V(H))} g_{A}(e) \phi(e)-\sum_{e^{\prime} \in \Gamma^{-}(V(H))} g_{A}\left(e^{\prime}\right) \phi\left(e^{\prime}\right) .
$$

Then we have the following Stokes' Theorem like formula.

Proposition. For a subdigraph $H$ of a labeled digraph $\left(D, g_{D}\right)$, we have

$$
\int_{H} \delta_{g_{D}} \phi=\int_{\partial H} \phi \text { for } \phi \in C^{1}(D)
$$

Proof. As the loops of $D$ give no contributions to the sums in the formula, we may assume that $D$ has no loops.

$$
\begin{aligned}
\int_{H} \delta_{g_{D}} \phi & =\sum_{v \in V(H)} g_{V}(v) \delta_{g_{D}} \phi(v) \\
& =\sum_{v \in V(H)}\left(\sum_{\substack{e \in A(D) \\
\alpha(e)=v}} g_{A}(e) \phi(e)-\sum_{\substack{e^{\prime} \in A(D) \\
\omega\left(e^{\prime}\right)=v}} g_{A}\left(e^{\prime}\right) \phi\left(e^{\prime}\right)\right) .
\end{aligned}
$$

For $e \in V(H)$, as the term $g_{A}(e) \phi(e)$ appears with the ' + ' sign in the summation of $\alpha(e) \in$ $V(H)$ and with the '-' sign in the summation of $\omega(e) \in V(H)$, the terms $g_{A}(e) \phi(e)$ cancel each other and disappear in the summation. Thus the summation of the last formula is taken 
for $e$ with the ' + ' $\operatorname{sign}$ for $\alpha(e) \in V(H), \omega(e) \notin V(H)$, and with the '-' sign for $\omega(e) \in$ $V(H), \alpha(e) \notin V(H)$. It then follows that

$$
\begin{aligned}
\int_{H} \delta_{g_{D}} \phi & =\sum_{e \in \Gamma^{+}(V(H))} g_{A}(e) \phi(e)-\sum_{e^{\prime} \in \Gamma^{-}(V(H))} g_{A}\left(e^{\prime}\right) \phi\left(e^{\prime}\right) \\
& =\int_{\partial H} \phi .
\end{aligned}
$$

A non-empty proper full subdigraph $K$ of a digraph $D$ is called a (+)-subdigraph (resp. $(-)$-subdigraph) if $\Gamma^{-}(V(K))=\emptyset\left(\operatorname{resp} . \Gamma^{+}(V(K))=\emptyset\right)$. Recall the definition of admissible functions on a digraph $D$ (cf. [7]). We call a function $f: V(D) \rightarrow \boldsymbol{R}$ admissible if every minimal (+)-subdigraph contains a vertex $v$ with $f(v)>0$, and every minimal $(-)$-subdigraph contains a vertex $w$ with $f(w)<0$. Here "minimal" means the usual set theoretical sense, that is, being non-empty and containing no non-empty proper $(+)$-subdigraphs (resp. (-)-subdigraphs). In case $D$ has no $( \pm)$-subdigraphs, any function $f$ with $f(v)>0$ and $f(w)<0$ for some $v, w \in V(D)$ or $f \equiv 0$ is called admissible.

A digraph $D$ is said to be strongly connected if there is a directed path from $u$ to $v$ for every distinct vertices $u, v \in V(D)$. If $D$ is not strongly connected, then there are $u$ and $v \in$ $V(D)$ so that there is no directed path from $u$ to $v$. Set $W_{u}=\{w \in V(D) \mid$ there is a directed path from $u$ to $w$ \}. It is easy to see that $D\left[W_{u}\right]$, the subdigraph of $D$ generated by the vertices $W_{u}$, is a (-)-subdigraph. Thus we have the following (cf. [7]).

LEMMA 1. A digraph D is strongly connected if and only if D has no $( \pm)$-subdigraphs.

Now we recall some relevant facts on foliations (see [3] for generalities on foliations and [11] for differential geometric aspects of foliations). Let $(M, \mathcal{F})$ be a transversely oriented codimension-one foliation $\mathcal{F}$ of a closed oriented manifold $M$. The transverse orientation of $\mathcal{F}$ determines a vector field $N$ on $M$ transverse to $\mathcal{F}$. A compact domain $C \subset M$ is called foliated if $C$ is a union of leaves of $\mathcal{F}$. A foliated compact domain $C$ is said to be $(+$ )-fcd (resp. $(-)$-fcd) if $N$ points outwards (resp. inwards) everywhere on $\partial C$ (cf. [8], [9]). We obtain, in a unique way, a digraph $\Gamma(M, \mathcal{F})$ from $(M, \mathcal{F})$, and from an arbitrarily given digraph $D$, we can construct a transversely oriented codimension-one foliation of a closed oriented manifold $(M, \mathcal{F})$ such that $D=\Gamma(M, \mathcal{F})$. Indeed, we have

THEOREM O1 ([9]). Let $(M, \mathcal{F})$ be as above. For each $(M, \mathcal{F})$ there exist a digraph $\Gamma(M, \mathcal{F})$ and a nice transverse embedding $\psi: \Gamma(M, \mathcal{F}) \rightarrow(M, \mathcal{F})$. Furthermore, for each arc $e \in A(D), \psi(\operatorname{Int}(e))$ intersects each compact leaf of $\mathcal{F}$ at most once.

THEOREM O2 ([9]). For any digraph $D$, there is a foliated manifold $(M, \mathcal{F})$ so that $D=\Gamma(M, \mathcal{F})$.

In these theorems, a vertex $v \in V(D)=V(\Gamma(M, \mathcal{F}))$ corresponds to a compact foliated domain $C_{v} \subset M$ and an $\operatorname{arc} e=(u, v) \in A(\Gamma(M, \mathcal{F}))$ to a compact leaf $L \in \mathcal{F}$ contained in $C_{u} \cap C_{v}$ with $\psi(e) \cap L \neq \emptyset$. The head $\omega(e)$ and the tail $\alpha(e)$ of an arc $e$ are determined 
by the direction of $N$ so that $N$ goes from $C_{\alpha(e)}$ to $C_{\omega(e)}$ along $L$. By the above construction, $( \pm)$-fcd's correspond to $( \pm)$-subdigraphs, respectively.

Let $f$ be a smooth function on $M$. We call $f$ admissible if there is a Riemannian metric $g$ on $M$ such that $-f$ coincides with the mean curvature function of $\mathcal{F}$ with respect to $g$ (see Walczak [13] or Oshikiri [5], [6]). A characterization of admissible functions, which was conjectured by Walczak and proved affirmatively by the author (see Oshikiri [8]), is the following.

THEOREM O3 ([9]). Let $\mathcal{F}$ be a transversely oriented codimension-one foliation of a closed connected oriented manifold $M$. Assume that $\mathcal{F}$ contains at least one $(+)-f c d$. Then $f$ is admissible if and only if $f(x)>0$ somewhere in any minimal $(+)-f c d$ and $f(y)<0$ somewhere in any minimal $(-)-f c d$. In case $\mathcal{F}$ contains no $(+)$-fcd's, any smooth function $f$ with $f(x)>0$ and $f(y)<0$ for some $x, y \in M$ or $f \equiv 0$ is admissible.

For a smooth function $h$ on $M$ with a volume element $d V$, define a function $\Gamma_{d V}(h)$ : $V(D) \rightarrow \boldsymbol{R}$ by

Then we have

$$
\Gamma_{d V}(h)(v)=\int_{D_{v}} h d V \quad \text { for } v \in V(D) .
$$

THEOREM O4 ([9]). For a smooth function $f$ on $M$, the following two conditions are equivalent.

(1) $f$ is admissible on $(M, \mathcal{F})$.

(2) There is a volume element $d V$ on $M$ so that $\Gamma_{d V}(f)$ is admissible on $\Gamma(M, \mathcal{F})$.

3. Proof and Remark. Let $D=(V(D), A(D))$ be a finite digraph and $(M, \mathcal{F})$ be a codimension-one foliation with $\Gamma(M, \mathcal{F})=D$ obtained in Theorem O2. To prove the theorem, we need the following.

LEMma 2. For any admissible function $f: V(D) \rightarrow \boldsymbol{R}$ there is a Riemannian metric $g$ of $M$ such that $\Gamma_{d V(M, g)}(h)=f$, where $d V(M, g)$ is the volume element of the Riemannian manifold $(M, g)$ and $-h$ is the mean curvature function of $\mathcal{F}$ with respect to the Riemannian metric $g$.

ProOF. Fix an arbitrary volume form $d V$ on $M$. For each $v \in V(D)$, choose a smooth function $k_{v}$ on $D_{v}$ with $\operatorname{supp}\left(k_{v}\right) \subset \operatorname{Int}\left(D_{v}\right)$ and $\int_{D_{v}} k_{v} d V=f(v)$. Define $k: M \rightarrow \boldsymbol{R}$ by $k(x)=k_{v}(x)$ for $x \in D_{v}, v \in V(D)$. As $k_{v}$ is smooth on $D_{v}$ and $\operatorname{supp}\left(k_{v}\right) \subset \operatorname{Int}\left(D_{v}\right), k$ is a smooth function on $M$ such that $\Gamma_{d V}(k)=f$. Furthermore, as $f$ is admissible on $D, k$ is also admissible on $M$ by Theorem O4. Thus, there is a Riemannian metric $\bar{g}$ of $M$ so that $-k$ is the mean curvature function of $\mathcal{F}$ with respect to $\bar{g}$. We deform $k$ and $\bar{g}$ into $h$ and $g$ so that $\Gamma_{d V(M, g)}(h)=f$. To do this, recall the following fact (see [4], Lemma 3 (ii), where the term $H^{\prime}=e^{-2 \psi} H$ should be corrected by $H^{\prime}=e^{-\psi} H$ ):

If $g|T \mathcal{F} \otimes T M=\bar{g}| T \mathcal{F} \otimes T M$ and $g(X, Y)=e^{2 \rho} \bar{g}(X, Y)$ for $X$ and $Y$ orthogonal to $\mathcal{F}$, then $h=e^{-\rho} k$, where $-h$ (resp. $-k$ ) is the mean curvature function of $\mathcal{F}$ with respect to the metric $g$ (resp. $\bar{g}$ ). 
By this change of the metric, as $d V(M, g)=e^{-\rho} d V(M, \bar{g})$, it follows that $\int_{D} h d V(M, g)=\int_{D} e^{-2 \rho} k d V(M, \bar{g})$. For each $v \in V(D)$, choose a suitable function $\rho_{v}$ on $D_{v}$ with $\operatorname{supp}\left(\rho_{v}\right) \subset \operatorname{Int}\left(D_{v}\right)$ and deform the metric $\bar{g}$ on $D_{v}$ so that $\int_{D_{v}} h d V(M, g)=f(v)$. By setting $\rho(x)=\rho_{v}(x)$ for $x \in D_{v}, v \in V(D)$, we have the desired Riemannian metric of $M$.

Proof of Theorem. Assume that $f$ is admissible. We shall show that there is a labelling $g_{D}$ of $D$ so that $f=\delta_{g_{D}}$. By Lemma 2, there is a Riemannian metric $g$ of $M$ such that $\Gamma_{d V}(h)=f$, where $d V$ is the volume element of the Riemannian manifold $(M, g)$ and $-h$ is the mean curvature function of $\mathcal{F}$ with respect to the Riemannian metric $g$. Let $N$ be the unit vector field on $M$ orthogonal to $\mathcal{F}$ such that the orientation coincides with the transverse orientation of $\mathcal{F}$. Then it is well-known that $\operatorname{div}_{g}(N)=h$.

Now, define a labelling $g_{D}=\left(g_{V}, g_{A}\right)$ of $D$ by

$$
g_{V}(v)=1 \quad \text { and } \quad g_{A}(e)=\operatorname{Area}\left(L_{e}, g \mid L_{e}\right),
$$

where $L_{e}, e=(u, v) \in A(D)$, is the unique leaf in $D_{u} \cap D_{v}$ intersecting with $\psi(e)$ (cf. Theorem O1), and Area $\left(L_{e}, g \mid L_{e}\right)$ is the volume of the Riemannian manifold $\left(L_{e}, g \mid L_{e}\right)$. Set $\partial^{+} D_{v}=\left\{\right.$ compact leaves $L \subset \partial D_{v}$ with $N$ pointing outwards on $\left.L\right\}$ and $\partial^{-} D_{v}=\{$ compact leaves $L \subset \partial D_{v}$ with $N$ pointing inwards on $\left.L\right\}$. Then, for each $v \in V(D)$, we have

$$
\int_{D_{v}} h=\int_{D_{v}} \operatorname{div}_{g}(N)=\sum_{L \in \partial^{+} D_{v}} \operatorname{Area}(L)-\sum_{L^{\prime} \in \partial^{-} D_{v}} \operatorname{Area}\left(L^{\prime}\right) .
$$

This implies, for $v \in V(D)$, that

$$
\begin{aligned}
f(v) & =\Gamma_{d V}(h)=\int_{D_{v}} h \\
& =\sum_{L \in \partial^{+} D_{v}} \operatorname{Area}(L)-\sum_{L^{\prime} \in \partial^{-} D_{v}} \operatorname{Area}\left(L^{\prime}\right) \\
& =\sum_{e \in \Gamma^{+}(v)} g_{A}(e)-\sum_{e^{\prime} \in \Gamma^{-}(v)} g_{A}\left(e^{\prime}\right) \\
& =\delta_{g_{D}} 1 .
\end{aligned}
$$

Thus, $g_{D}$ is the desired one.

Conversely, Assume that $f=\delta_{g_{D}} 1$ for some labelling $g_{D}$. Then, by Proposition and $\partial D=\emptyset$, we have

$$
\int_{D} f=\int_{D} \delta_{g_{D}} 1=\int_{\partial D} 1=0 .
$$

This implies that $f \equiv 0$ or $f(u)>0$ and $f(v)<0$ for some $u, v \in V(D)$. If $D$ is strongly connected, then, as $D$ has no $( \pm)$-subdigraphs, $f$ is, by definition, admissible. Assume $D$ is not strongly connected and $H$ is a minimal (+)-subdigraph. As $H$ is a (+)-subdigraph, we 
have $\Gamma^{+}(V(H)) \neq \emptyset$ and $\Gamma^{-}(V(H))=\emptyset$. It follows that

$$
\int_{H} f=\int_{H} \delta_{g_{D}} 1=\int_{\partial H} 1=\int_{\Gamma^{+}(V(H))} 1>0,
$$

which means that $f(v)>0$ for some $v \in V(H)$. For a minimal (-)-subdigraph $K$, by the same argument, we have $\int_{K} f<0$, which means that $f(w)<0$ for some $w \in V(K)$. Thus, $f$ is, by definition, admissible. This completes the proof.

REMARK. From the construction of the labelling in the proof, we get the following result corresponding to Theorem $\mathrm{O} 2$ for a labeled digraph $\left(D, g_{D}\right)$ :

For any labeled digraph $\left(D, g_{D}\right)$, there are a foliated manifold $(M, \mathcal{F})$ and a Riemannian metric $g$ of $M$ such that $D=\Gamma(M, \mathcal{F})$ and the labelling $g_{D}$ is given as in the proof of the theorem from the Riemannian metric $g$.

In [10], the author studied a relation of Cheeger constant and strong connectivity of finite digraphs. As a corollary to the theorem, we give a labeled digraph version of this result. To this end, define Cheeger constant $\operatorname{ch}(D, g)$ for a labeled finite digraph $(D, g)$ by

$$
\begin{aligned}
& \operatorname{ch}(D, g) \\
& \quad=\min \left\{\frac{\sum_{e \in \partial H} g_{A}(e)}{\sum_{v \in V(H)} g_{V}(v)} \mid H \text { is a subdigraph of } D \text { with } \sum_{v \in V(H)} g_{V}(v) \leq \frac{1}{2} \sum_{v \in V(D)} g_{V}(v)\right\} .
\end{aligned}
$$

Recall that a digraph is weakly connected if the underlying graph is connected.

COROLlaRY. Let $D$ be a weakly connected finite digraph. Then, $D$ is strongly connected if and only if there is a labelling $g_{D}$ of $D$ such that $\operatorname{ch}\left(D, g_{D}\right)>\max _{v \in V(D)}\left|\delta_{g_{D}} 1(v)\right|$.

PROOF. If $D$ is strongly connected, then the function $f \equiv 0$ is admissible, and, by the theorem, there is a labelling $g_{D}$ of $D$ such that $f=\delta_{g_{D}} 1 \equiv 0$. As $\operatorname{ch}\left(D, g_{D}\right)>0$, it follows that $\operatorname{ch}\left(D, g_{D}\right)>\max _{v \in V(D)}\left|\delta_{g_{D}} 1(v)\right|=0$.

Conversely, assume that there is a labelling $g_{D}$ of $D$ such that $\operatorname{ch}\left(D, g_{D}\right)>$ $\max _{v \in V(D)}\left|\delta_{g_{D}} 1(v)\right|$. If $D$ is not strongly connected, then, by Lemma 1 , there is a (+)subdigraph $H$. We may assume that $\sum_{v \in V(H)} g_{V}(v) \leq 1 / 2 \cdot \sum_{v \in V(D)} g_{V}(v)$ (if not, consider the (-)-subdigraph $D \backslash H)$. As $\partial H=\Gamma^{+}(H)$, by Proposition, it follows that

$$
\sum_{e \in \partial H} g_{A}(e)=\int_{\partial H} 1=\int_{H} \delta_{g_{D}} 1<\operatorname{ch}\left(D, g_{D}\right) \cdot \int_{H} 1 \text {. }
$$

Thus, we have

$$
\operatorname{ch}\left(D, g_{D}\right) \leq \frac{\sum_{e \in \partial H} g_{A}(e)}{\sum_{v \in V(H)} g_{V}(v)}=\frac{\int_{H} \delta_{g_{D}} 1}{\int_{H} 1}<\frac{\operatorname{ch}\left(D, g_{D}\right) \cdot \int_{H} 1}{\int_{H} 1}=\operatorname{ch}\left(D, g_{D}\right),
$$

which is a contradiction, and this completes the proof. 


\section{REFERENCES}

[1] N. BIGgS, Algebraic graph theory, Cambridge Math. Lib., Cambridge University Press, Cambridge, 1993.

[2 ] J. A. Bondy, Basic Graph Theory: Paths and Circuits, Chapter 1 in 'Handbook of Combinatorics' Vol. 1 (ed. by R. L. Graham, M. Grötschel and L. Lovász), 3-110, North-Holland, Amsterdam, 1995.

[ 3 ] G. Hector And U. Hirsch, Introduction to the geometry of foliations: Part B, Aspects Math. E3, Friedr. Vieweg \& Sohn, Braunschweig, 1983.

[ 4 ] G. OSHIKIRI, On codimension-one foliations of constant curvature, Math. Z. 203 (1990), 105-113.

[ 5 ] G. OshikiRI, Mean curvature functions of codimension-one foliations, Comment. Math. Helv. 65 (1990), 79-84.

[ 6 ] G. OshikiRI, Mean curvature functions of codimension-one foliations II, Comment. Math. Helv. 66 (1991), $512-520$.

[ 7 ] G. OsHIKIRI, A remark on oriented graphs, Interdiscip. Inform. Sci. 2 (1996), 85-88.

[ 8 ] G. OsHIKIRI, A characterization of the mean curvature functions of codimension-one foliations, Tôhoku Math. J. 49 (1997), 557-563.

[ 9 ] G. OSHIKIRI, Codimension-one foliations and oriented graphs, Tôhoku Math. J. 51 (1999), 227-236.

[10] G. OshikiRI, Cheeger constant and connectivity of graphs, Interdiscip. Inform. Sci. 8 (2002), 147-150.

[11] P. TondeuR, Geometry of Foliations, Monogr. Math. 90, Birkhäuser Verlag, Basel, 1997.

[12] H. UraKawa, Laplacian and Networks, Shokabo, Tokyo, 1996.

[13] P. WALCZAK, Mean curvature functions for codimension-one foliations with all leaves compact. Czechoslovak Math. J. 34 (1984), 146-155.

FACULTY OF EDUCATION

IWATE UNIVERSITY

18-33, UEDA 3-CHOME

MORIOKA, 020-8550

JAPAN

E-mail address: oshikiri@iwate-u.ac.jp 